\title{
Development of a Novel Standard Type of Gel Engineering Materials via Simple Bulk Polymerization*
}

\author{
Jin GONG**, Yosuke WATANABE**, Yusuke WATANABE**, Ruri HIDEMA***, \\ Muhammad Hasnat KABIR**,**** and Hidemitsu FURUKAWA** \\ ${ }^{*}$ Soft \& Wet matter Engineering Laboratory (SWEL), Department of Mechanical Systems Engineering, \\ Graduate School of Science and Engineering, Yamagata University, \\ 4-3-16 Jonan, Yonezawa, Yamagata, Japan, 992-8510 \\ E-mail: furukawa@yz.yamagata-u.ac.jp \\ ***Organization of Advanced Science and Technology, Kobe University, \\ 1-1 Rokkodai, Nada, Kobe, Hyogo, Japan, 657-8501

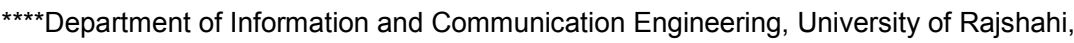 \\ Rajshahi, Bangladesh, 6205
}

\begin{abstract}
Gels are soft and wet materials, which have unique properties such as low surface friction, material permeability, and biocompatibility. These superior properties of gels can possibly be applied to develop novel artificial blood vessels and can also be used at knee joints. However, the mechanical properties of the gels have not been clarified and controlled so far due to the lack of standard type of gels for mechanical engineering research. Here we try to develop a novel type of gel named the standard gel engineering materials (standard GEM) via simple bulk polymerization, i.e. dry synthesis without any solvent. In the numerous previous studies of gels, almost all gels have been prepared by solution polymerization, i.e. wet synthesis with various solvents. The mechanical properties of the wet-synthesized gels, however, strongly depend on the concentrations of the monomer, crosslinker, initiator, and accelerator in pre-gel solutions. Therefore, it is difficult to control the mechanical properties of gels causing rough reproducibility of mechanical experiments. In the present paper, the simple bulk polymerization is proposed as another candidate to create a standard GEM. The key point of the present work is to use the liquid type of highly reactive monomer, which plays the role of solvent in preparation, and thus we do not have to consider both the ratio concentrations of the monomer and the accelerator to the solvent in preparation. In this case we can focus only on the ratio concentration of the crosslinker to the monomer. This ratio concentration is directly related to the contour length of the polymer chain between the crosslinking points. We have found that the mechanical properties of the dry-synthesized GEM depend hardly on the ratio concentration of the initiator to the monomer, but mainly on that of the crosslinker. The Young's modulus is simply, well described as a power function of the crosslinker concentration. We hope this dry-synthesized GEM will be useful as another novel standard sample for mechanical engineering research.
\end{abstract}

Key words: Soft and Wet Materials, Gels, Mechanical Properties, Standardization, Bulk Polymerization

${ }^{*}$ Received 20 Sep., 2012 (No. 12-0388) [DOI: 10.1299/jmmp.7.455]

Copyright (C) 2013 by JSME 


\section{Introduction}

Gels are soft and wet materials, made from a large amount of solvent and a small amount of three-dimensional opened network structures of chain-like molecules in the network structure. The gels, thus, sometime become hard like a plastic or a metal and they possess excellent properties, for example, low surface friction, shock absorbance, materials permeability, softness, high ductility, shape memory, and biocompatibility. These properties have been applied to develop novel smart materials and devices, such as smart medical tape ${ }^{(1)}$ ultrahigh ductile materials, ${ }^{(2)}$ intelligent push button, ${ }^{(3)}$ and smart optical lens. ${ }^{(4)}$ These functional gels, further, are conveniently fabricated by using optical 3D gel printer. ${ }^{(5)}$ However, the mechanical properties of the gels have not been clarified and controlled so far due to the lack of a standard type of gels for mechanical engineering. In the numerous previous studies of the gels, those were started after the landmark studies done by the MIT group of Prof. Toyoichi Tanaka in 1970s, ${ }^{(6-9)}$ almost all the gels have been prepared by solution polymerization, i.e. wet synthesis with various solvents. The mechanical properties of the wet-synthesized gels, however, strongly depend on the concentrations of the monomer, crosslinker, initiator, and accelerator in pre-gel solutions. It causes notorious difficulty to control the mechanical properties of gels and the reproducibility of mechanical experiments is not enough good, which was studied by the detailed experiment of the equilibrium swelling using the dynamic light scattering for systematically wet-synthesized polyacrylamide gels. ${ }^{(10-12)}$ The rough reproducibility makes it difficult to use the gels as industrial materials. It is considered that the reason of this ill reproducibility is mainly related to the solvent of water. The water usually contains oxygen, which behaves as inhibitor for free radical polymerizations. The existence of the oxygen slows down the rate of propagation and causes the incompleteness of the polymerization reaction. The low concentration of monomer in dilute solution also causes the incompleteness of the polymerization reaction. Polyacrylamide gel, one of standard hydrogels, was prepared by standard solution polymerization. ${ }^{(9-12)}$ The monomers, crosslinker and initiator were dissolved in distilled degassed water and then the gelation was done. Here we propose the bulk polymerization in order to make the gelation undergo more simply and finally to obtain gels having reproducible properties. That is, there is no need to use the solvent in the bulk polymerization. The bulk polymerization has possibly two additional advantages. One is environment-friendly since no solvent is used. The other is simplicity since we do not have to consider the monomer concentration in preparation. In the present study, the standard type of gel engineering materials (standard GEM) with reproducible properties is prepared by using the bulk polymerization and systematically studies about water content, refractive index, and mechanical properties. We try to confirm whether the standard GEM will possibly be used as industrial materials.

\section{Experimental}

\subsection{Materials}

Liquid $N, N$-dimethylacrylamide (DMAAm) was purchased from TOKYO CHEMICAL INDUSTRY CO., LTD., Japan. White needle crystal of $N$, $N^{\prime}$-methylenebis (acrylamide) (MBAA) and white powder of $\alpha$-ketoglutaric acid ( $\alpha$-keto) were purchased from Wako Pure Chemical Industries ltd., Japan. All the above chemicals were used as received. The Mill-Q (made by Millipore's apparatus) water was used for all the experiment as distilled water. 
2.2 Bulk polymerization
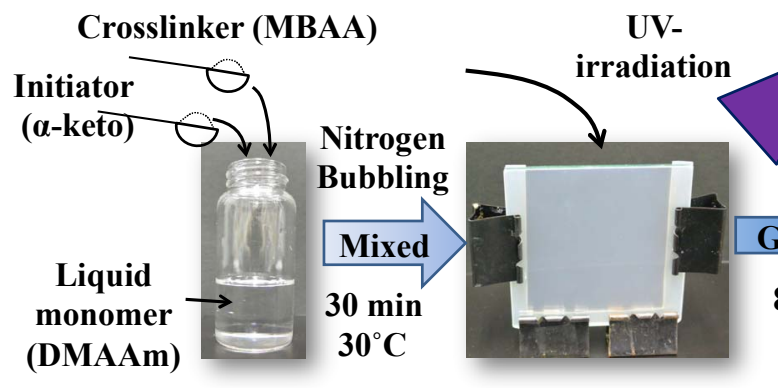

UV-

Figure 1. Simple dry preparation of the DMAAm gels without solvent.

Table 1. The components of the gels prepared in the present study.

\begin{tabular}{|c|c|c|c|c|c|c|c|c|c|c|c|c|}
\hline $\begin{array}{c}\text { Sample } \\
\text { No. }\end{array}$ & 1 & 2 & 3 & 4 & 5 & 6 & 7 & 8 & 9 & 10 & 11 & 12 \\
\hline $\begin{array}{c}\text { DMAAm } \\
{[\mathrm{mol}]}\end{array}$ & 1.0 & 1.0 & 1.0 & 1.0 & 1.0 & 1.0 & 1.0 & 1.0 & 1.0 & 1.0 & 1.0 & 1.0 \\
\hline $\begin{array}{c}\text { MBAA } \\
{[\text { mol\%] } *}\end{array}$ & 0.02 & 0.02 & 0.02 & 0.05 & 0.05 & 0.05 & 0.1 & 0.2 & 0.2 & 0.2 & 0.4 & 0.4 \\
\hline $\begin{array}{c}\alpha \text {-keto * } \\
{[\text { mol\%] }}\end{array}$ & 0.01 & 0.04 & 0.1 & 0.01 & 0.04 & 0.1 & 0.1 & 0.01 & 0.04 & 0.1 & 0.1 & 0.1 \\
\hline $\begin{array}{c}\text { Water * } \\
{[\text { mol\%] }}\end{array}$ & 0.0 & 0.0 & 0.0 & 0.0 & 0.0 & 0.0 & 0.0 & 0.0 & 0.0 & 0.0 & 0.0 & 150 \\
\hline
\end{tabular}

* The ratio of the chemical to DMAAm.
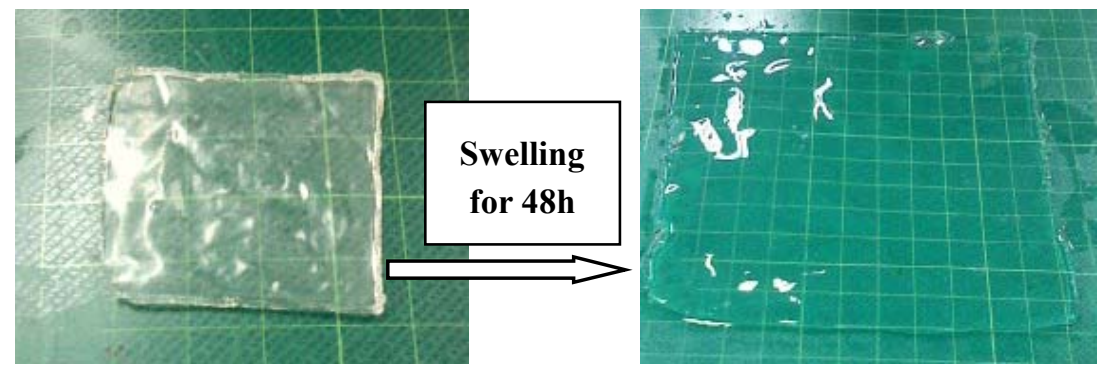

Figure 2. A gel became about 3.5 times larger in volume after swelling.

The standard GEM samples were synthesized by using the DMAAm as monomer, the MBAA as intercross-linker, and the $\alpha$-keto as photo initiator through free-radical polymerization. The monomer of the DMAAm also plays the role of solvent because the DMAAm is a liquid monomer at room temperature and both the MBAA and the $\alpha$-keto are soluble in DMAAm. The preparation scheme is shown in Figure 1. The MBAA and the $\alpha$-keto were added to the DMAAm and stirred for 30 minutes with nitrogen bubbling to remove oxygen. The pregel solution was poured into the mold. The pregel solution was irradiated by UV lamp (the peak wavelength is $365 \mathrm{~nm}$ ) for 8 hours and the gelation was completed. In the present study, 11 gels were prepared by varying the amount of monomer, crosslinker and initiator, as listed in Table 1 (No. 1-11). The prepared gels were soaked into a large amount of the Mill-Q water for more than 48 hours to achieve swelling equilibrium. 
The volume of the swollen gel became several times larger comparing to that of the as-prepared gel as shown in Figure 2. The volume change depends on the components in preparation.

\subsection{Solution polymerization in water}

For comparison, a gel sample was synthesized by solution polymerization in water using DMAAm, MBAA, and $\alpha$-keto. Both the MBAA and the $\alpha$-keto were added to the DMAAm and stirred for 30 minutes. The Mill-Q water was added into and stirred for 30 minutes. The pregel solution was poured into the mold and was irradiated by UV lamp (the peak wavelength is $365 \mathrm{~nm}$ ) for 8 hours. The composites of the gel were also listed in Table 1 (No. 12).

\subsection{Characterization}
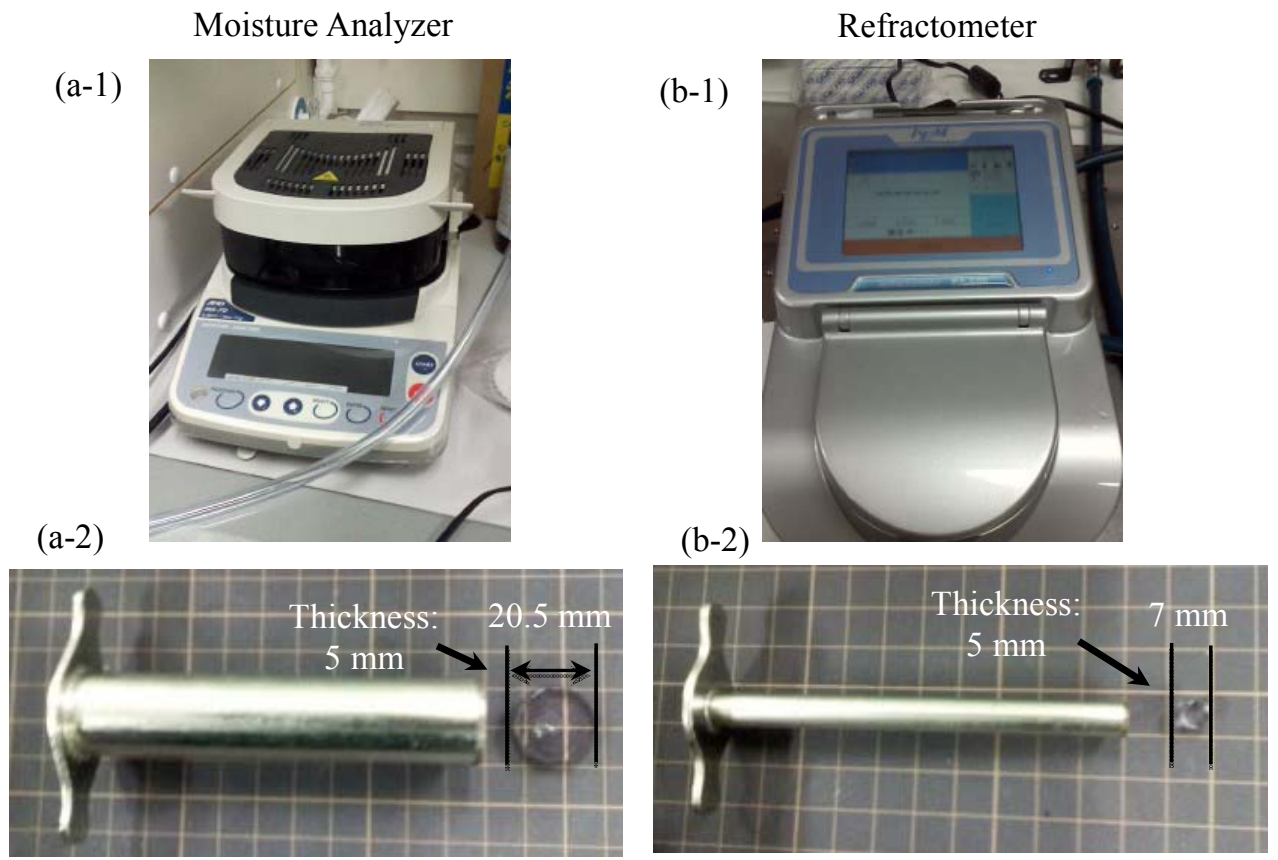

Figure 3. The moisture analyzer, the refractometer, and the dimensions of samples.

The water content $(W)$ was measured by a moisture analyzer (A\&D Company, Limited, Saitama, Japan). The refractive index $(n)$ was recorded using a refractometer RA-600 (Kyoto Electronics Manufacturing Co., Ltd., Kyoto, Japan). The apparatuses are shown in Figure 3 (a-1) and (b-1). Sample sizes for $W$ measuring are $20.5 \mathrm{~mm}$ in diameter and $5 \mathrm{~mm}$ in thickness by using the baller shown in Figure 3 (a-2). Sample sizes for $n$ measuring are 7 $\mathrm{mm}$ in diameter and $5 \mathrm{~mm}$ in thickness by using the baller shown in Figure 3 (b-2). The tensile test was performed using a universal testing machine STA-1150 (ORIENTEC Company, Limited, Tokyo, Japan) (Figure 4 (a)). Dumbbell specimen (JIS K6251 Dumb-bell test pieces No. 8) shown in Figure 4 (b) was used for tensile testing. The tensile test was carried out under the crosshead speed of $100 \mathrm{~mm} / \mathrm{min}$ at room temperature. 

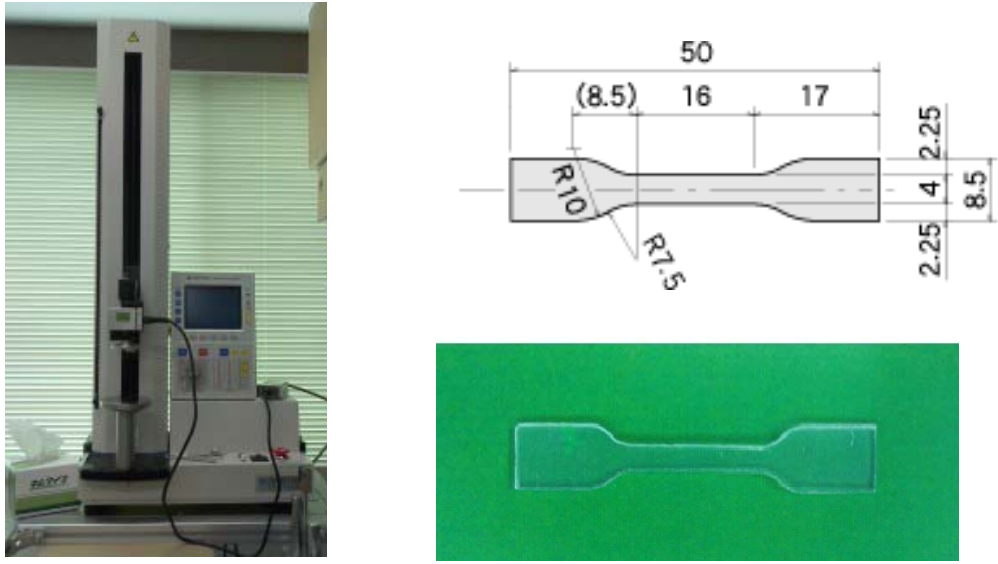

Figure 4. The universal testing machine and the dimensions of dumbbell specimen.

\section{Results and Discussion}

The gelation was not completed for Sample No. 1, 4 and 8 due to the least amount $(0.01 \mathrm{~mol} \%)$ of initiator $\alpha$-keto. The most probable reason is that the initiator was too little to induce radical initiation sufficiently. When the amount of initiator $\alpha$-keto was more than $0.01 \mathrm{~mol} \%$, the gelation was completed smoothly and transparent gels were obtained. The $n$ and $W$ of the gels polymerized at $0.1 \mathrm{~mol} \%$ of $\alpha$-keto (No. 3, 6. 9, 10, 11 and 12) were measured and the results are shown in Table 2. The gel samples prepared by bulk polymerization (No. 3, 6, 9, 10, 11) present lower standard deviation in $n$ and $W$, while the No. 12 gel prepared by solution polymerization in water shows higher standard deviation. It indicates that the gels prepared by bulk polymerization have more stability in $n$ and $W$ compared to that of gel prepared in water. The amount of initiator $\alpha$-keto is required more than $0.01 \mathrm{~mol} \%$ for the complete gelation.

Table 2. Measurement results of $\boldsymbol{n}$ and $\boldsymbol{W}$

\begin{tabular}{|l|l|c|c|c|c|c|c|}
\hline \multicolumn{2}{|c|}{ Sample No. } & 3 & 6 & 9 & 10 & 11 & 12 \\
\hline \multirow{2}{*}{$\mathrm{n}[-]$} & Average & 1.3335 & 1.3338 & 1.3368 & 1.3362 & 1.3368 & 1.3362 \\
\cline { 2 - 8 } & $\begin{array}{l}\text { Standard } \\
\text { deviation }\end{array}$ & 0.0003 & 0.0005 & 0.0007 & 0.0006 & 0.0007 & 0.0006 \\
\hline \multirow{2}{*}{$\begin{array}{l}\mathrm{W} \\
{[\mathrm{wt} \%]}\end{array}$} & Average & 97.3 & 94.9 & 90.3 & 90.3 & 72.6 & 76.7 \\
\cline { 2 - 8 } & $\begin{array}{l}\text { Standard } \\
\text { deviation }\end{array}$ & 0.103 & 0.253 & 0.746 & 0.349 & 0.647 & 0.949 \\
\hline
\end{tabular}




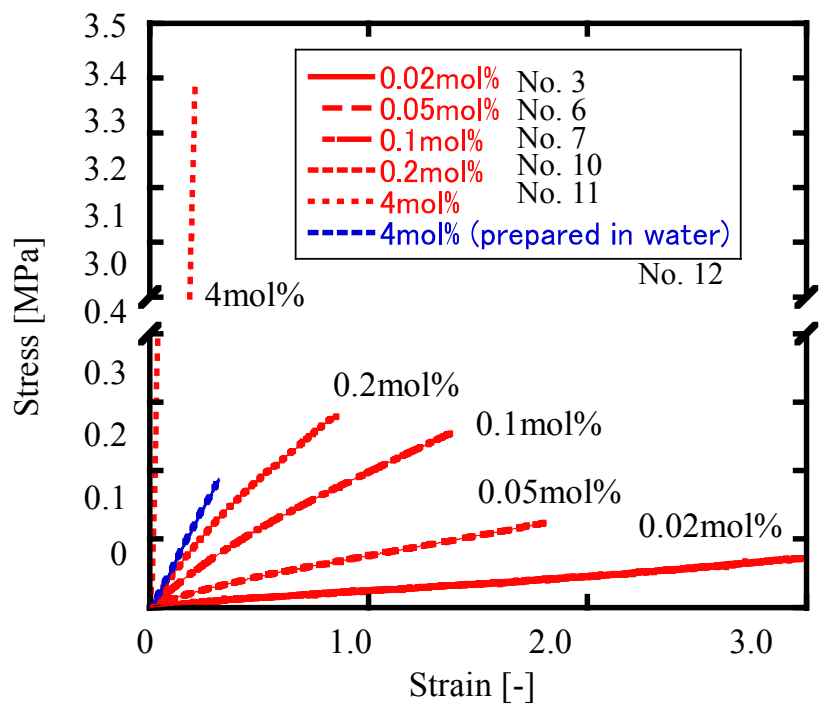

Figure 5. The stress-strain curves of gels prepared by bulk polymerization (red lines) and by solution polymerization in water (blue line).

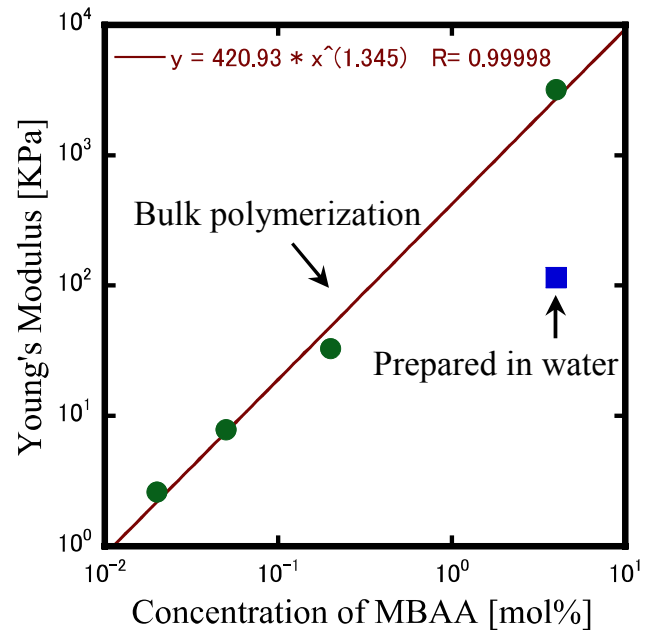

Figure 6. The linear relationship between the Young's modulus of the DMAAm gels and the concentration of the crosslinker MBAA.

The tensile tests were performed for the six samples listed in Table 2. Figure 5 shows their stress-strain curves. It is found that the tensile strength decreases and the stretch increases with the increasing of the amount of crosslinker MBAA from $0.02 \mathrm{~mol} \%$ to $4 \mathrm{~mol} \%$. The gels polymerized by bulk polymerization (No. 11) and by solution polymerization in water (No. 12) at the same amount $4 \mathrm{~mol} \%$ of MBAA were compared. The No. 11 gel shows the highest stress of $3.4 \mathrm{MPa}$, about 20 times higher from the No. 12 gel, while both samples show almost similar strain. It is very clear that the bulk polymerization is quite good to prepare the standard gels having better mechanical properties. Figure 6 shows the curve of the Young's modulus $E$ vs. the concentration $c$ of the crosslinker MBAA. It can be seen clearly that the Young's modulus of GEM prepared by bulk polymerization is much higher (around 20 times) than that of the sample prepared by solution polymerization in water. It can also be seen that the Young's modulus of GEM increases with the increasing of the concentration of crosslinker MBAA in the bulk polymerization case. It is additionally noted that this behavior is possibly fitted to the 
power-law function, ${ }^{(13)} E=E_{0} c^{\alpha}$, where $E_{0}$ is a coefficient and $\alpha$ is an exponent. The data is well fitted with $E_{0}=420 \mathrm{kPa}$ and $\alpha=1.35$. The power-law relation between Young's modulus and concentration of crosslinker makes it possible to provide an evaluating in mechanical properties based on the amount of crosslinker. It implies that the properties of the standard GEM prepared by bulk polymerization are capable of being expressed only by the function of $c$ theoretically. This is quite helpful and convenient to standardize the gel materials in the industry field.

Finally here we like to add some other information about the bulk polymerization. Recently we have succeeded in developing transparent type of shape memory gels (SMG) and their applications using this technique. ${ }^{(1,3,4)}$ The transparent SMG is undoubtedly novel since the previous SMGs are completely turbid and whiten, i.e. they are not transparent, as far as we know. ${ }^{(14)}$ This is also important achievement via the bulk polymerization. The transparency as well as the above-mentioned good mechanical properties is critically important to apply these gels to develop optical devices and add to the rich clear appearance of gel products. Thus we believe that the bulk polymerization is quite simple but will be a breakthrough technology for the standardization of gels.

\section{Conclusion}

Using bulk polymerization, the standard GEM having stable properties and homogeneous structure can be produced easily. This will promote the rapid progress of mechanical engineering research. The crosslinker ratio to the monomer $(c[\mathrm{~mol} \%])$ is the only factor, which is greatly influenced in the properties of the standard GEM in the bulk polymerization. This means it is possible to regulate the standard GEM by noting them in symbols like "DMAAm- $0.2 \mathrm{~mol} \% \mathrm{MBAA}$ gel". The range of $c$ in the bulk polymerization is quite wider $(0 \sim$ several dozens mol\%) compared with the solution polymerization in water ( $0 \sim$ several mol\%). The properties of the standard GEM prepared by bulk polymerization are capable of being expressed only by the function of $c$ theoretically. Precise and quantitative study on the standard GEM produced by bulk polymerization will spread in our next work by making full use of the above knowledge.

\section{Acknowledgments}

This study was partly supported by the Industrial Technology Research Grant Program in 2009 (Project ID: 09A25003a) from the New Energy and Industrial Technology Development Organization (NEDO) of Japan, and partly supported by the Grant-in-Aid for Scientific Research (B) (Project No.: 22350097) from the Japan Society for the Promotion of Science (JSPS). This work was also partly supported by the national project named "Green Tribology Innovation Network", in area of Advanced Environmental Materials, Green Network of Excellence (GRENE), sponsored by the Ministry of Education, Culture, Sports, Science and Technology (MEXT) in Japan. This work was also partly supported by the Yonezawa City Grant in 2012 and the Yamagata University Stand-up Grant for New Teachers in 2012 .

\section{References}

(1) Amano, Y., Hidema, R., Gong, J., Furukawa, H., "Creation of Shape-memory Gels with Inter-crosslinking Network Structure”, Chemistry Letters, Vol. 41, No. 10, (2012), pp. 1029-1031.

(2) Takada, G., Hidema, R., Furukawa, H., "Ultrahigh Ductile Gels Having Inter-Crosslinking Network (ICN) Structure", e-Journal of Surface Science and Nanotechnology, Vol. 10, (2012), pp. 346-350. 
(3) Harada, S., Hidema, R., Gong, J., Furukawa, H., "Intelligent Button Developed with Smart Soft and Wet Materials", Chemistry Letters, Vol. 41, No. 10, (2012), pp. 1047-1049.

(4) Yokoo, T., Hidema, R., Furukawa, H., "Smart Lenses Developed with High-Strength and Shape Memory Gels", e-Journal of Surface Science and Nanotechnology, Vol. 10, (2012), pp. 243-247.

(5) Muroi, H., Hidema, R., Gong, J., Furukawa, H., "Development of Optical 3D Gel Printer for Fabricating Free-Form Soft \& Wet Industrial Materials and Evaluation of Printed Double-Network Gels", Journal of Solid Mechanics and Materials Engineering, Vol. 7, No. 2, (2013), pp. 163-168.

(6) Tanaka, T., Hocker, L. O., Benedek, G. B., "Spectrum of Light Scattered from a Viscoelastic Gel”, The Journal of Chemical Physics, Vol. 59, No. 9, (1973), pp. 5151-5159.

(7) Tanaka, T., Ishiwata, S., Ishimoto, G., "Critical Behavior of Density Fluctuations in Gels", Physical Review Letters, Vol. 38, No. 14, (1977), pp. 771-774.

(8) Tanaka, T., "Collapse of Gels and the Critical Endpoint", Physical Review Letters, Vol. 40, No. 12, (1978), pp. 820-823.

(9) Tanaka, T., Sato, E., Hirokawa, Y., Hirotsu. S., Peetermans, J., "Critical Kinetics of Volume Phase Transition of Gels," Physical Review Letter, Vol. 55, No. 22, (1985), pp. 2455-2458.

(10)Furukawa, H., "Effect of Varying Preparing Concentration on the Equilibrium Swelling of Polyacrylamide Gels”, Journal of Molecular Structure, Vol. 554, (2000), pp. 11-19.

(11)Furukawa, H., Horie, K., Nozaki, R., Okada, M., "Swelling-induced Modulation of Static and Dynamic Fluctuations in Polyacrylamide Gels Observed by Scanning Microscope Light Scattering”, Physical Review E, Vol. 68, (2003), pp. 031406-1-14.

(12)Furukawa, H., Hirotsu, S., "Dynamic Light Scattering from Static and Dynamic Fluctuations in Inhomogeneous Media,” Journal of Physical Society of Japan, Vol. 71, No. 12, (2002), pp. 2873-2880.

(13) Watanabe, Y., Maekawa, K., Kabir, M. H., Hidema, R., Gong, J., Furukawa, H., "Strucural Analysis and Mechanical Properties of Dry-Synthesis Gels", Journal of Solid Mechanics and Materials Engineering, Vol. 7, No. 2, (2013), pp. 224-227.

(14) Mitsumata, T., Gong, J.P., Osada, Y., "Shape Memory Functions and Motility of Amphiphilic Polymer Gels", Polymers for Advanced Technologies, Vol. 12, No. 1-2, (2001), pp. 136-150. 